\title{
Post-marketing safety surveillance for inactivated and live-attenuated Japanese encephalitis vaccines in China, 2008-2013
}

\author{
Wu Wendi ${ }^{a, b, 1}$, Liu Dawei ${ }^{a, 1}$, Li Keli ${ }^{a^{*}}$, Pekka Nuorti ${ }^{b}$, Hanna M. Nohynek ${ }^{c}$, Xu Disha ${ }^{a}$, Ye \\ Jiakai $^{a}$, Zheng Jingshan ${ }^{a}$, Wang Huaqing,*.
}

a National Immunization Programme, Chinese Center for Disease Control and Prevention

b School of Health Sciences, FIN-33014 UNIVERSITY OF TAMPERE, FINLAND

c National Institute for Health and Welfare THL Helsinki Finland

$1 \mathrm{Wu}$ Wendi and Liu Dawei contributed equally to this work.

* Corresponding authors:Wang Huaqing and Li Keli. Tel:+86 10 63171892(Wang)/831663931(Li), E-mail address: wanghq@ chinacdc.cn(Wang)/likl@chinacdc.cn(Li)

\begin{abstract}
Introduction: Two types of Japanese encephalitis (JE) vaccines, inactivated JE vaccine (JE-I) and liveattenuated JE vaccine (JE-L), are available and used in China. In particular, one JE-L, produced by a domestic manufacturer in China, was prequalified by WHO in 2013. We assessed the safety of JE vaccines in China during 2008-2013 using the Chinese National Adverse Events Following Immunization Information System (CNAEFIS) data.
\end{abstract}

Methods: We retrieved AEFI reporting data about JE vaccines from CNAEFIS, 2008-2013, examined demographic characteristics of AEFI cases, and used administrative data on vaccine doses as denominator to calculate and compare crude reporting rates. We also used disproportionality reporting analysis between JE-I and JE-L to assess potential safety signals.

Results: A total of 34,879 AEFIs related with JE-I and JE-L were reported, with a ratio of male to female as $1.3: 1 ; 361(1.0 \%)$ cases were classified as serious. JE vaccines were administered concurrently with one or more other vaccines in 13,592 (39.0\%) of cases. The overall AEFI reporting rates were 214.4 per million vaccination doses for JE-L and 176.9 for JE-I (rate ratio [RR]: 1.2, 95\% confidence interval [CI]: 1.1-1.3) in 2010-2013. Febrile convulsions (FC) following JE-I was found as a signal of disproportionate reporting (SDR). However, there was no significant difference between the reporting rates of FC of JE-I and JE-L (0.3 per million vaccination doses for JE-L, 0.4 for JE-I, $\mathrm{p}=0.05$ ).

Conclusions: While our analysis did not find apparent safety concern of JE vaccines in China, further study should consider consider JE-I vaccines and febrile convulsion, and taking more sensitive methods to detect signals.

Key words: Vaccine safety, adverse events following immunization, febrile convulsion, Japanese encephalitis vaccine, disproportionality analysis, surveillance system, China 


\section{Introduction}

Japanese encephalitis (JE) is a mosquito-borne acute viral infection of the central nervous system caused by a flavivirus [1]. JE is the most important cause of vaccine-preventable viral encephalitis in nearly all Asian countries, whether temperate, subtropical, or tropical, and has expanded into new areas through the importation of infected-mosquito vectors. Currently, an estimated 3 billion people living in 24 countries, mainly in the South-East Asia and Western Pacific Regions are considered at risk of JE [2]. The inactivated JE vaccine (JE-I) was developed in China and has been used since the 1970s and live-attenuated vaccine (JE-L) was in the beginning of the 1990s [1]. Since 2007, JE vaccines were included into the Expanded Program on Immunization (EPI) in the mainland of China [1]. With the decline of number of JE disease in China, the public became more concern about the adverse events following JE vaccination currently. The safety of JE vaccines manufactured in China and abroad was evaluated in previous clinical and post-marketing studies [3-7]. The vaccine safety review of JE vaccines by World Health organization (WHO) were found to have acceptable safety profiles, and data from multiple studies (including multicenter randomized controlled trail and randomized trials) had shown the same conclusion [2,8-9]. However, The JE vaccine used in China were mainly produced by domestic manufacturers, and a JE-L product was prequalified by WHO in 2013, which was the first Chinese-produced vaccine to be prequalified by WHO. Limited data are available on the safety of JE after its inclusion into the Chinese EPI and consequently its large-scale use. Concurrently with the inclusion of JE vaccines into EPI, the Chinese national adverse event following immunization (AEFI) information system (CNAEFIS), a passive post-marketing vaccine safety surveillance system, was also expanded to cover the entire country after a 3-year pilot study [10,11]. With 6 years of information collection, there were valuable AEFI data in the CNAEFIS which could provide some evidence for evaluation of vaccine safety in China. Also, using the passive surveillance data, the disproportionality analysis, which was first used in signal detection of drug safety, could also be necessary to generate signals, especially when the causality of the specific events and vaccination has not been well known. We conducted a study to analyze AEFI data for both JE-I and JE-L vaccines in CNAEFIS from 2008 to 2013 to understand and compare the vaccine safety profiles of these 2 vaccines. 


\section{Methods}

\section{Vaccination schedules of JE vaccines in China [10]}

In the mainland of China, both JE-I and JE-L have been included in National Immunization Program (NIP) vaccinations since 2007. For JE-L, a 2-dose schedule is used: at 8 and 24 months of age, with at least 3 months' interval. For JE-I, a 4-dose schedule is used ( 2 doses at 8 months with at least 7-10 day intervals, and subsequent doses at 2 and 6 years of age).

\section{Vaccination doses of JE vaccines}

All the vaccines should be used in Vaccination clinics, which was approved by local government and supervised by local Center for diseases control and prevention (CDC). Vaccination doctors or nurses collected information of vaccination doses and reported to the county CDCs monthly. The county CDCs report the data to municipal CDCs who report to provincial CDCs. China CDC collects administered vaccination data from all provincial CDCs $[3,13]$.

Before 2010, only doses of vaccines provided by the government for free were reported. Therefore, complete data on all JE vaccination doses given during 2008-2009 are not available. Since 2010, however, the vaccination information system collected all vaccination doses administered in the vaccination clinics, enabling the analysis of the reporting rates. As the denominator information only included the number of vaccine doses, without information on age or sex, the rates of AEFI for specific population groups by age or gender could not be calculated.

\section{CNAEFIS}

The online CNAEFIS is administered by the Chinese Center for Disease Control and Prevention (CCDC). After two pilot studies, it was expanded to cover all 31 provinces in the mainland of China in 2008 [10]. The pilot studies included a passive surveillance system for AEFI in 10 provinces and an enhanced AEFI surveillance system in 5 counties. CNAEFIS is operated in accordance with China's national AEFI guidelines [12]. According to this guidance, CNAEFIS became the official AEFI information system and was to be owned and maintained by China CDC [10].

An AEFI case is defined as a reaction or an event occurring after vaccine administration that is suspected to be related to the vaccination. AEFI surveillance and reporting covers all vaccines marketed in the mainland of China [10].

\section{Reporting and Investigation [10-13]}

Healthcare facilities, vaccination clinics, Centers for Disease Control and Prevention (CDCs) at all 4 administrative levels, adverse drug reaction monitoring agencies (ADRs), and vaccine manufacturers are required by law to report suspected AEFIs. The public or the guardian (parents) can notify any of the above authorized reporters to report an AEFI. Cases are investigated by local, county-level CDCs, which are responsible for completing AEFI Case Reporting Cards and submitting the data to online CNAEFIS. Once the information is entered, it can be viewed by all administrative levels of CDCs and ADRs. Based on the address, name and birthday of the child, vaccines, and vaccination dates, duplicate reports are identified and potential multiple reports are combined into one case. 
Investigation is required for all AEFIs, except common adverse reactions with a clear diagnosis (e.g., fever; redness, swelling, and induration on the injection site). For deaths, serious AEFIs, AEFI clusters, and AEFIs of significant public concern that are suspected to be related to vaccination, prefectural or provincial CDCs must immediately organize an AEFI expert panel for investigation upon receiving CNAEFIS reports.

\section{Serious and non-serious AEFIs [13]}

Serious AEFI is defined as an event that is causing a potential risk to the health/life of a recipient leading to prolonged hospitalization, disability/incapacity, congenital abnormalities/ birth defects or death. In CNAEFIS, it include, but are not limited to, allergic shock, allergic laryngeal edema, allergic purpura, thrombocytopenic purpura, localized allergic necrotic reaction (Arthus reaction), febrile convulsion, epilepsy, brachial neuritis, polyneuritis, Guillain-Barre syndrome, encephalopathy, encephalitis and meningitis, syncope, toxic shock syndrome, and systemic purulent infection.

\section{Data analysis}

We analyzed AEFI reports submitted during 2008-2013, for subjects vaccinated with JE-L or JE-I. In CNAEFIS, a maximum 3 suspected vaccines can be reported at the same time in a single report. JE vaccines listed as the first, second, or third suspected vaccine were all included. When more than one symptom was reported for a case, only the main symptom or the most serious diagnosis was recorded in CNAEFIS.

The age and sex distribution and clinical diagnoses were described, and crude AEFI reporting rates per million doses given were calculated. Since there is no information on whether the vaccines were NIP vaccine or not in AEFI cases, the incidence of NIP vaccine or voluntary vaccination could not be estimated during the study year.

We used disproportionality analysis of data mining algorithms to compare the frequency of reports for JE-L and JE-I to detect any signal of disproportionate reporting (SDR) [14]. Disproportionality analysis identifies AEFIs that were more frequent than expected and relies on the principle that when a SDR is identified for a specific vaccine, this event (or diagnosis) is reported relatively more frequently in association with this specific vaccine than all the other vaccine in the database. Three disproportionality analysis methods were applied: the proportional reporting ratio (PRR) [15-16], Bayesian confidence propagation neural network (BCPNN) [17], and empirical Bayesian (EB) data mining [18-20]. Disproportionality analysis were based on a $2 * 2$ contingency table similar to a case-control study or cohort study [21]. Calculations were performed using R (version i386 3.2.3), and the PhViD package were used in analysis.

Since disproportionality analysis required "vaccine and diagnosis" as a pair, cases without confirmed clinical diagnosis were excluded. For the cases in which diagnosis included common minor adverse reactions, with a mix of symptoms such as fever, local redness, local swelling, and other minor local or systemic symptoms, "common reaction" was used as a single diagnosis. Cases who had received concurrent vaccines were excluded. 


\section{Results}

\section{AEFI following JE-L and JE-I}

A total of 34,879 AEFI cases associated with JE vaccines were collected by CNAEFIS, 2008- 2013; 95.2\% (33,186) cases were related to JE-L. JE vaccines were administered concurrently with one or more other vaccines in 13,592 (39.0\%) of cases (39.9\% for JE-L and 19.9\% for JE-I, respectively). Both for JE-L and JE-I, the most common concurrently administered vaccine was measlescontaining vaccines, with a proportion of $24.8 \%$ for JE-L (8226 cases in 33186 JE-L-related cases) and $17.5 \%$ for JE-I (297 cases in 1693 JE-I-related cases).

JE-L was listed as the first suspected vaccine in 23,627 (71.2\% of the JE-L-associated AEFI cases). JE-I was the first suspected vaccine in 1357 (80.2\% of JE-I-associated cases) ( $p<0.05)$.

There were more cases in males than in females, with a sex ratio of $1.3: 1$. More cases occurred in $\leq 1$ years of age, with $66.4 \%$ of JE-L and $60.8 \%$ of JE-I (Table 1). Of all 34,879 AEFI cases, 361 (1.0\%) AEFI cases were defined as serious.

There were 146.7 million vaccination doses collected of JE vaccines from 2010-2013, in which 95.1\% (139.5 million doses) was JE-L. Since both JE-L and JE-I could be used as NIP vaccines and voluntary vaccines, among all JE vaccination doses, $91.5 \%$ (134.3 million doses) were used as NIP vaccines, including 133.1 million of JE-L and 1.2 million of JE-I. Using the doses administered from 2010 to 2013 as denominators, the overall reporting rates of AEFIs per million were 214.4 for JE-L and 176.9 for JE-I (RR: 1.2, 95\% CI: 1.1-1.3). the annual reporting rates increased substantially from 2010 to 2013 (Table 2). During 2010-2013, 271 serious AEFIs were reported. The overall reporting rates of serious AEFIs were 1.8 per million doses for JE-L and 2.8 per million doses for JE-I (RR: 0.7, 95\% CI: 0.4-1.0).

\section{Clinical diagnosis of AEFIs}

Of the 29,831 non-serious AEFIs, (86.4\%) were diagnosed as common and minor adverse reactions, such as fever, local redness, and swelling.

Among serious AEFIs, the most frequently reported clinical diagnosis were febrile convulsion (132, $36.6 \%)$, thrombocytopenic purpura $(39,10.8 \%)$, encephalitis and meningitis $(29,8.0 \%)$, HenochSchönlein purpura $(28,7.8 \%)$, and anaphylactic shock $(25,6.9 \%)$. (Table 3$) 22$ death cases were reported during the study period, only 4 cases were classified as related to vaccination due to anaphylactic shock.

\section{Disproportionality analysis}

A total of 20,988 AEFIs with complete information on vaccine and diagnosis were included in the disproportionality analysis. All three methods, PRR, EB, and BCPNN, suggested JE-I and febrile convulsion as the suspected SDRs (Table 3). For JE-L, there was no diagnosis with disproportionally higher reporting.

Based on the results of Table 4, using the administered doses from 2010-2013 as the denominator, the estimated reporting rates of febrile convulsion after JE-L (as the only vaccine suspected) and JE-I (as the only vaccine suspected) were calculated: 0.3 per million doses for JE-L and 0.4 per million doses for JE-I $(\mathrm{p}=0.5)$. 


\section{Discussion}

We reviewed reported AEFIs after JE-L and JE-I in CNAEFIS by 3 levels of analysis: a description of the characteristics of AEFIs, disproportionate reporting between JE-L and JE-I and estimated reporting rates. The serious AEFIs only accounted for $1 \%$ of all reported AEFIs, and there was also no statistical difference between JE-L and JE-I in the reporting rates of serious AEFI in all 20102013.However in 2012, the reporting rates of serious AEFI following JE-L were lower than JE-I. The increasing trend in the reporting rates of AEFI following the immunization of JE-L and JE-I may be related to the improved reporting to CNAEFIS during 2008 to 2013. The increased trends were similar to the reporting rates of all AEFIs during the same period; the reporting rates of all AEFIs in CNAEFIS of China continuously increased from 2010 to 2013 [22-25].

In previous CNAEFIS data analysis, only JE vaccines were included in the study [20-23]. However, in our study, at least one third of AEFIs had other concurrently administered vaccines. In addition, in the $\leq 1$-year group, which was the target group of EPI, an in AEFI for JE-L, more than half the cases were related to JE-L in combination with other vaccines. In these situations, it is difficult to determine the responsible vaccine for some adverse events, such as allergic reactions. Since concurrent vaccination could not be avoided, we included all AEFIs related to JE in the CNAEFIS database.

The gender distribution of JE-L and JE-I AEFIs cases was similar to all other vaccines [22-24], as there were more male than female cases. Most reported cases were $\leq 1$ year old, consistent with the China Immunization schedule for EPI vaccines. Most reported AEFIs were relatively mild and selflimiting, with a diagnosis of common and minor adverse reactions, including fever, local redness and swelling, and local induration. Besides the mild and self-limiting adverse reactions, nearly $97 \%$ of cases recovered, and only 22 death cases were reported during the study period.

Currently, there are several JE vaccines used in China. About 8 domestic manufacturers in China produce JE-L and JE-I. In the mainland of China, since 1968, JE-I vaccines (Hamster kidney cell inactivated vaccine) were manufactured domestically. The mass vaccination period of JE was after 1989, the year JE-L was developed and manufactured by Chengdu institute of biological products company, and until 2001, the yield of JE-L was more than 200 million doses in all. Following the positive assessment by the WHO of China's vaccine National Regulatory Authority in 2011 [25] and with the development of a vaccine manufactory in China [26], JE-L was the first vaccine in China to be prequalified by the WHO [27]. Different JE vaccines have been used in other countries. For example, the United States (US) used an inactivated mouse brain-derived JE vaccine (JE-I) for travelers (most of them were adults) from 1992 to 2009. Japan used JE-I for children. Takahashi [4] reviewed the post-marketing surveillance of JE-I from Japan and the US. The total adverse events rate was 2.8 per 100,000 doses in Japan and 15.0 per 100,000 doses in the US.

JE-L was widely used in countries in the Western Pacific region and Asia (China, Korea, and India), but very limited data are available on the adverse effects of JE-L in these countries. An analysis of the post-marketing surveillance of JE-L from 2009-2012 by the Chinese National Center for Adverse Drug Reaction (ADR) Monitoring [2] showed that in 6024 AEFIs, only 70 were considered severe. The Global Advisory Committee on Vaccine Safety (GACVS) of the WHO reviewed these data and noted that although there was no evidence of a safety signal, the number of events recorded in the AEFI reporting system was low, given that $>70$ million doses of the vaccine have been administered [25]. 
One of the primary goals of AEFI passive surveillance is to detect vaccine safety signals and generate hypotheses for further studies. Using vaccination doses as a denominator, AEFI incidence rates are calculated. Through a comparison with historic data and published studies, emerging vaccine safety signals are detected. However, since serious AEFIs are very rare, and it is difficult to determine the background incidence rate, in recent decades, researchers on pharmacovigilance have applied data mining methods in the detection of drug adverse events' signals on vaccines [28]. One of the main analysis methods is disproportionality analysis. In the US, PRR [15-16], which was also used in the UK Yellow Card Scheme (YCS) (the spontaneous reporting system in the UK), and the Gamma Poisson Shrinkage model (GPS), which was also noted as EB data mining, has been used to screen Vaccine Adverse Events Reporting System (VAERS) data and generate signals, including signals from seasonal influenza vaccines and from newly licensed vaccines [18-20]. The WHO Uppsala Monitoring Center (UMC) has used a similar data mining method, BCPNN, to detect safety signals $[17,29]$. These methods were not used as routine SDR screening in CNAEFIS, but we applied the methods to compare the SDR between JE-L and JE-I as supplementary analysis to evaluate the safety of JE in China. Febrile convulsions are the commonest type of seizure in children occurring in $2-5 \%$ of all children, $2-5 \%$ of young children in North America and Europe, and 6-9\% in Japan [30-31]. It usually occurs between 3 months and 5 years, with a peak incidence at 18 months [32], which was also the target age of vaccinations, especially for JE-L and JE-I. Generally, at least $50 \%$ of children who present with febrile convulsion will have no identified risk factors [33]. As elevated body temperature is frequently observed following immunization, and febrile seizures are the most common seizure disorder in infants and children, they are the most common type of non-epileptic seizure observed following immunization [33]. The signal of febrile convulsion has been found after measles-mumps-rubella combined vaccine (MMR), diphtheriatetanus-pertussis combined vaccine (DTP), and seasonal influenza vaccine in some countries, such as the US and Australia [34-37]. However, an increased risk of febrile convulsion after JE has rarely been reported. In a randomized trial with 26239 subjects, within 30 days follow-up, the incidence proportion of seizure was $0.1 \%$, and fever lasting more than 3 days was $2.7 \%$ in the group vaccinated with JE-L. There was no statistically significant difference between vaccinated and unvaccinated groups for seizure or fever lasting more than 3 days [38]. In our study, using the 3 data-mining methods, an SDR: JE-I and febrile convulsion was detected compare with JE-L. This indicated that there was a disproportionality in reports of febrile convulsion after JE-I compared to JE-L. However, when comparing the overall estimated reporting rates of febrile convulsion after both vaccines, there was no significant difference. In addition, febrile seizures after JE were less common than after measles-contained vaccines and varicella vaccines [36]. Therefore, the SDR of JE-I and febrile convulsion should be interpreted with caution.

CNAEFIS, as a national passive surveillance system, has its strengths and limitations. Even if it serves as a useful source for vaccination safety evaluation in China, the findings in CNAEFIS should be interpreted with caution. The natural limitations of passive surveillance include over- and under-reporting, biased reporting, and inconsistency in the quality and completeness of reports among others [37]. Meanwhile, the clinical diagnosis of AEFI cases varied in provinces of China, as we did not have a standard procedure or definition of AEFI diagnosis, and we did not use the standard definitions created by the Brighton Collaboration [39]. In our analysis, since CNAEFIS were case-based reporting system, and vaccination doses were collected based on summarized tables from vaccination clinics, these 2 data resources were not exact matched. There were no information on whether the vaccine used as NIP vaccine or voluntary vaccines in CNAEFIS, and no 
gender and age information on vaccination doses, all those related incidence could not be estimated using current data.

In conclusion, during the study period of 2008-2013, the majority of AEFIs following JE vaccines were minor and common adverse reactions, and there was no significant difference between the estimated reporting rates of serious AEFI following JE-I and JE-L. For serious AEFIs, more than $97 \%$ of cases recovered. We recommend consider further study to discern effects of concurrent vaccination with $\mathrm{JE}$ vaccines and take more sensitive methods to detect signals.

\section{References}

[1] Wang Huanyu, Li Yixing, Liang Xiaofeng, et al. Japanese Encephalitis in Mainland China. Jpn J Infect Dis 2009, 62:331-336.

[2] World Health Organization. Japanese Encephalitis Vaccines: WHO position paper-February 2015. WER 2015, 90(6): 69-88.

[3] Liu Yu, Lin Hualiang, Zhu Qi, et al. Safety of Japanese encephalitis live attenuated vaccination in post-marketing surveillance in Gyangdong, China, 2005-2012. Vaccine 2014, 32:1768-1773.

[4] Hiroshi Takahashi, Vitali Pool, Theodore F Tsai, et al. Adverse events after Japanese encephalitis vaccination: review of post-marketing surveillance data from Japan and the United States. Vaccine 2000,18: 2963-2969.

[5] Tetsuo Nakayama, Kazumasa Onoda. Vaccnie adverse events reported in post-marketing study of the Kitasato Institute from 1994-2004. Vaccnie 2007, 25: 570-576.

[6] H D Nothdurft, T Jelinek, A Marschang, et al. Adverse reactions to Japanese encephalitis vaccine in travelers. Journal of Infection 1996, 32: 119-122.

[7] Tom Solomon, Nguyen Minh Dung, Rachel Kneen, et al. Japanese encephalitis. J Neurol Neurosurg Psychiatry 2000;68:405-415.

[8] Chokephaibulkit K, Sirivichayakul C, Thisyakorn U, et al. Safety and immunogenicity of a single administration of live-attenuated Japanese encephalitis vaccine in previously primed 2- to 5-year-olds and naive 12- to 24-month-olds: multicenter randomized controlled trial. Pediatr Infect Dis J,2010;29(12):1111-1117.

[9] Liu ZL, Hennessy S, Strom BL, et al. Short-term safety of live attenuated Japanese encephalitis vaccine (SA14142): results of a randomized trial with 26,239 subjects. J Infect Dis,1997;176(5):1366-1369.

[10] Liu Dawei, Wu Wendi, Li Keli, et al. Surveillance of adverse events following immunization in China: Past,present, and future. Vaccine 2015, 33: 4041-4046.

[11] Liang Xiaofeng, Li Li, Liu Dawei, et al. Safety of Influenza A (H1N1) Vaccine in Postmarketing Surveillance in China. N Engl J Med 2011, 364: 638-47.

[12] China Ministry of Health, Food and Drug administration of China. National guideline for the surveillance of adverse events following immunization. Beijing: China Ministry of Health, Food and Drug administration of China; 2010.

[13] China Ministry of Health. Chinese Standard Procedures for Vaccination. Beijing: China Ministry of Health; 2005.

[14] Manfred Hauben, Jeffrey K Aronson. Defining 'Signal' and its subtypes in Pharmacovigilance based on a systematic review of previous definitions. Drug safety 2009, 32(2): 99-110. 
[15] Evans S.J.W., Waller P.C., Davis S. Use of Proportional reporting ratios (PRR) for signal generation from spontaneous adverse drug reaction reports. Pharmacoepidemiology and Drug Safety 2001; 10: 483-486.

[16] European Medicines Agency. Eudra Vigilance Expert Working Group. Guideline on the use of statistical signal detection methods in the Eudra Vigilance data analysis system. (EMEA/106464/2006 rev. 1) [Online]. Available from URL:

http://www.ema.europa.eu/docs/en_GB/document_library/Regulatory and_procedural_guideline/2009/11/WC5000114 34.pdf [Accessed 2015.8.11]

[17] Andrew Bate, Marie Lindquist, I Ralph Edwards, et al. A data mining approach for signal detection and analysis. Drug safety 2002, 25(6): 393-397.

[18] DuMouchel W. Bayesian data mining in large frequency tables, with an application to the FDA spontaneous reporting system (with discussion) [J]. Am Stat, 1999, 53(3):177-190.

[19] John Iskander, Vitali Pool, Weigong Zhou, et al. Data Mining in the US using the Vaccine Adverse Event Reporting System[J]. Drug Safety,2006,29(5):375-384.

[20] Barbara A. Slade, Laura Leidel, Claudia Vellozzi, et al. Postlicensure Safety Surveillance for Quadrivalent Human Papillomavirus Recombinant Vaccine. JAMA 2009; 302(7): 750-757.

[21] Marc suling, Iris Pigeot. Signal detection and monitoring based on longitudinal healthcare data, Pharmaceutics 2012, 4, 607-640.

[22] Wu Wendi, Liu, Dawei, Li Keli, et al. Analysis on Surveillance Data of Adverse Events Following Immunization in China, 2010. Chinese journal of Vaccine and Immunization 2012, 18(5): 385-397.

[23] Wu Wendi, Li Keli, Zheng Jingshan, et al. Analysis on Surveillance Data of Adverse Events Following Immunization in China, 2011. Chinese journal of Vaccine and Immunization 2013, 19(2): 97-109.

[24] Wu Wendi, Liu Dawei, Li Keli, et al. Analysis on Surveillance Data of Adverse Events Following Immunization in China, 2012. Chinese journal of Vaccine and Immunization 2014, 20(1): 1-12.

[25] GACVS/WHO. Global Advisory Committee on Vaccine Safety, 12-13 June 2013. WER, 2013,88(29):301-312.

[26] WHO. Available from URL:

(http://www.who.int/immunization_standards/vaccine_regulation/nra_china_functional/en/accessed 15.9.2015)

[27] WHO. Available from URL:

(http://www.who.int/mediacentre/news/releases/2013/japanese_encephalitis_20131009/en/ accessed 14.9.2015).

[28] Manfred Hauben, David Madigan, Charles M Gerrits. The role of data mining in pharmacovigilance.Expert Opin. Drug saf 2005, 4(5): 929-948.

[29] Stephenson WP, Hauben M. Data mining for signals in spontaneousreporting databases: proceed with caution. Pharmacoepidemiol Drug Saf2007;16(4):359-65.

[30] Azhar Daoud. Febrile convulsion: review and update. Journal of Pediatric Neurology 2004; 2(1): 9-14.

[31] Tonia Jones, Steven J. Jacobsen. Childhood febrile seizures: overview and implications. International journal of medical science 2007, 4(2): 110-114.

[32] C Waruiru, R Appleton. Febrile seizures: an update. Arch Dis Child 2004;89:751-756.

[33] Fernando Cendes, Raman Sankar. Vaccinations and febrile seizures. Epilepsia 2011, 52(Suppl. 3):23-25. 
[34] Yuelian Sun, Jakob Christensen, Anders Hviid, et al. Risk of Febrile Seizures and Epilepsy After Vaccination With Diphtheria, Tetanus, Acellular Pertussis, Inactivated Poliovirus, and Haemophilus Influenzae Type b. JAMA. 2012; 307(8):823-831.

[35] Z. Leroya, K. Broder, D. Menschik, et al. Febrile seizures after 2010-2011 influenza vaccine in young children, United States: A vaccine safety signal from the vaccine adverse event reporting system. Vaccine 2012, 30: 2020-2023.

[36] Steven J. Jacobsena, Bradley K. Ackerson, Lina S. Sy, et al. Observational safety study of febrile convulsion following first dose MMRV vaccination in a managed care setting. Vaccine 2009, 27: 4656-4661.

[37] Frederick Varricchio, John Iskander, Frank Destefano, et al. Understanding vaccine safety information from the vaccine adverse event reporting system. Pediatr Infect Dis J 2004; 23: 287-94.

[38] Liu Zheng-Le, Sean Hennessy, Brian L. Strom, et al. Short-Term safety for live attenuated Japanese encephalitis vaccine (SA14-14-2): results of a randomized trial with 26239 subjects. JID 1997, 176: 1366-1369.

[39] Jan Bonhoeffer, Katrin Kohl, Robert Chen, et al. The Brighton Collaboration: addressing the need for standardized case definitions of adverse events following immunization (AEFI). Vaccine 2002, 21: 298-302.

Table 1 Characteristics of AEFIs after JE-L and JE-I vaccination, China, 2008-2013

\begin{tabular}{|c|c|c|c|c|c|c|c|c|c|}
\hline \multirow[b]{3}{*}{ Serious } & \multirow[b]{3}{*}{ Serious } & \multicolumn{4}{|c|}{ JE-L } & \multicolumn{4}{|c|}{ JE-I } \\
\hline & & \multicolumn{2}{|c|}{$\begin{array}{c}\text { As most suspected } \\
\text { N=23627 (\%) }\end{array}$} & \multicolumn{2}{|c|}{$\begin{array}{c}\text { others } \\
\mathrm{N}=9559(\%)\end{array}$} & \multicolumn{2}{|c|}{$\begin{array}{c}\text { As most suspected } \\
\mathrm{N}=1357(\%)\end{array}$} & \multicolumn{2}{|c|}{$\begin{array}{c}\text { Others } \\
\mathbf{N}=\mathbf{3 3 6}(\%)\end{array}$} \\
\hline & & 244 & 1.03 & 66 & 0.69 & 51 & 3.76 & 0 & 0.00 \\
\hline & Non-serious & 23383 & 98.97 & 9493 & 99.31 & 1306 & 96.24 & 336 & 100.00 \\
\hline \multirow[t]{2}{*}{ Gender } & Female & 13444 & 56.90 & 5358 & 56.05 & 768 & 56.60 & 190 & 56.55 \\
\hline & Male & 10183 & 43.10 & 4201 & 43.95 & 589 & 43.40 & 146 & 43.45 \\
\hline \multirow[t]{3}{*}{$\begin{array}{l}\text { Age } \\
\text { group }\end{array}$} & $\leq 1 \mathrm{yr}$ & 13306 & 56.32 & 8712 & 91.14 & 710 & 52.32 & 320 & 95.24 \\
\hline & $2-6 y r s$ & 10036 & 42.48 & 825 & 8.63 & 511 & 37.66 & 14 & 4.17 \\
\hline & $\geq 7 \mathrm{yrs}$ & 285 & 1.21 & 22 & 0.23 & 136 & 10.02 & 2 & 0.60 \\
\hline
\end{tabular}

* There were at most three suspected vaccines were reported in one AEFI cases, the reporters will put one vaccine as the most suspected vaccines. JE as most suspected means JE was most suspected related to adverse events in the reports. 
Table 2 Number and estimated AEFI reporting rates of after JE-L and JE-I by severity* of AEFI, China, 2010-2013

\begin{tabular}{|c|c|c|c|c|c|c|c|c|c|c|}
\hline \multirow[b]{3}{*}{ AEFI } & \multicolumn{4}{|c|}{ JE-L } & \multicolumn{4}{|c|}{ JE-I } & \multicolumn{2}{|c|}{ Rate ratios } \\
\hline & \multicolumn{2}{|c|}{ Non-serious } & \multicolumn{2}{|c|}{ Serious } & \multicolumn{2}{|c|}{ Non-serious } & \multicolumn{2}{|c|}{ Serious } & \multirow{2}{*}{$\frac{\text { Non-serious }}{\left(95^{\circ}\right.}$} & Serious \\
\hline & $\begin{array}{l}\text { No. of } \\
\text { cases }\end{array}$ & $\begin{array}{l}\text { Reporting } \\
\text { rates** }\end{array}$ & $\begin{array}{l}\text { No. of } \\
\text { cases }\end{array}$ & $\begin{array}{l}\text { Reporting } \\
\text { rates }\end{array}$ & $\begin{array}{l}\text { No. of } \\
\text { cases }\end{array}$ & $\begin{array}{l}\text { Reporting } \\
\text { rates }\end{array}$ & $\begin{array}{l}\text { No. of } \\
\text { cases }\end{array}$ & $\begin{array}{l}\text { Reporting } \\
\text { rates }\end{array}$ & & CI) \\
\hline 2010 & 2428 & 75.97 & 43 & 1.35 & 96 & 33.99 & 5 & 1.77 & $\begin{array}{r}2.23 \\
(1.83-2.76) \\
1.35\end{array}$ & $\begin{array}{r}0.74 \\
(0.32-2.17) \\
0.85\end{array}$ \\
\hline 2011 & 5816 & 168.51 & 41 & 1.19 & 102 & 124.86 & 1 & 1.22 & $\begin{array}{r}(1.12-1.65) \\
0.82\end{array}$ & $\begin{array}{r}(0.19-19.85) \\
0.38\end{array}$ \\
\hline 2012 & 8495 & 240.25 & 69 & 1.95 & 756 & 294.80 & 13 & 5.07 & $\begin{array}{r}(0.76-0.88) \\
1.16\end{array}$ & $\begin{array}{r}(0.22-0.72) \\
2.37\end{array}$ \\
\hline 2013 & 12908 & 343.11 & 98 & 2.60 & 308 & 295.79 & 1 & 0.96 & $(1.04-1.30)$ & $(0.54-54.72)$ \\
\hline $\begin{array}{l}2010- \\
2013\end{array}$ & 29647 & 212.60 & 251 & 1.80 & 1262 & 174.15 & 20 & 2.76 & $\begin{array}{r}1.22 \\
(1.15-1.29)\end{array}$ & $\begin{array}{r}0.65 \\
(0.42-1.05)\end{array}$ \\
\hline
\end{tabular}

*The severity of AEFI were classified according to national AEFI guidelines. Serious AEFIs include, but are not limited to, allergic shock, allergic laryngeal edema, allergic purpura, thrombocytopenic purpura, localized allergic necrotic reaction (Arthus reaction), febrile convulsion, epilepsy, brachial neuritis, polyneuritis, Guillain-Barre syndrome, encephalopathy, encephalitis and meningitis, syncope, toxic shock syndrome, and systemic purulent infection. **Reporting rates: per million vaccination doses given 
Table 3 Clinical Diagnosis of serious AEFI after the immunization of JE-L and JE-I, China, 2008-2013

\begin{tabular}{|c|c|c|c|c|c|c|}
\hline \multirow[b]{2}{*}{ Clinical diagnosis } & \multicolumn{4}{|c|}{ JE-L } & \multirow{2}{*}{\multicolumn{2}{|c|}{$\begin{array}{c}\text { JE-I* } \\
\text { First } \\
\text { suspected, } \\
\mathrm{N}=51(\%)\end{array}$}} \\
\hline & \multicolumn{2}{|c|}{$\begin{array}{c}\text { First } \\
\text { suspected, } \\
\mathrm{N}=\mathbf{2 4 4}(\%) \\
\end{array}$} & \multicolumn{2}{|c|}{$\begin{array}{c}\text { Not first } \\
\text { suspected, } \\
\mathrm{N}=66(\%)\end{array}$} & & \\
\hline Febrile Convulsion & 83 & 34.02 & 16 & 24.24 & 33 & 64.71 \\
\hline Thrombocytopenic Purpura & 22 & 9.02 & 15 & 22.73 & 2 & 3.92 \\
\hline Encephalitis and Meningitis & 23 & 9.43 & 5 & 7.58 & 1 & 1.96 \\
\hline Henoch-Schönlein Purpura & 18 & 7.38 & 6 & 9.09 & 4 & 7.84 \\
\hline Anaphylactic Shock & 21 & 8.61 & 1 & 1.52 & 3 & 5.88 \\
\hline Apsychia & 11 & 4.51 & 4 & 6.06 & 5 & 9.80 \\
\hline Seizure & 16 & 6.56 & 1 & 1.52 & 1 & 1.96 \\
\hline Laryngeal Edema & 4 & 1.64 & 1 & 1.52 & 1 & 1.96 \\
\hline $\begin{array}{l}\text { Acute Disseminated Encephalomyelitis } \\
\text { (ADEM) }\end{array}$ & 5 & 2.05 & 0 & 0.00 & 0 & 0.00 \\
\hline Arthus Reaction & 3 & 1.23 & 1 & 1.52 & 0 & 0.00 \\
\hline Guillain Barre Syndrome (GBS) & 1 & 0.41 & 1 & 1.52 & 0 & 0.00 \\
\hline Others & 37 & 15.16 & 15 & 22.73 & 1 & 1.96 \\
\hline
\end{tabular}

*For JE-I, There was no cases coded as serious AEFIs when administered in combination with other vaccines.

Table 4 Suspected SDR of JE using 3 DPA methods, China, 2008-2013

\begin{tabular}{ll}
\hline Methods (Criteria) & Results \\
\hline PRR (Lower limit of 95\% CI of & JE-I- Febrile Convulsion \\
PRR $>1$ \& $>3$ ) & $=1.59$ \\
& JE-I- Febrile Convulsion \\
EB (EB05 $>2$ \& $>3$ ) & EB05 $=3.27$ \\
& JE-I- Febrile Convulsion \\
BCPNN (Lower limit of 95\% CI of & Lower limit of $95 \%$ CI of IC $=1.64$ \\
IC $>1$ \& $>3)$ & \\
\hline
\end{tabular}

